\title{
An Optimized-Hierarchy-Aided Approximate Log-MAP Detector for MIMO Systems
}

\author{
Jos Akhtman, Member, IEEE, Andreas Wolfgang, Member, IEEE, \\ Sheng Chen, Senior Member, IEEE, and Lajos Hanzo, Fellow, IEEE
}

\begin{abstract}
In this paper we propose a novel Space Division Multiplexing (SDM) detection method. The proposed technique constitutes a list search method and may be regarded as an advanced extension of the Sphere Decoder (SD). Our method may be employed in the so-called over-loaded scenario, where the number of transmit antenna elements exceeds that of the receive antenna elements. Furthermore, it is suitable for highthroughput, non-constant modulus modulation schemes, such as 16 and 64-QAM. We introduce a series of optimization rules which facilitate a substantial reduction in computational complexity. More specifically, we demonstrate that the method proposed, which we refer to as the Soft-output OPtimized HIErarchy (SOPHIE)-aided SDM detector exhibits the near-optimum performance of Log-MAP SDM detector in all considered scenarios. The associated computational complexity, which we control using two complexity-control parameters, is substantially lower than that imposed by all previously proposed methods.
\end{abstract}

Index Terms-Bell Labs layered space-time (BLAST), fading channels, maximum likelihood (ML) detection, smart antennas, sphere decoding, turbo codes, wireless communications.

\section{INTRODUCTION}

$\mathbf{T}$ HE relevant information-theoretical analysis predicts [1] that substantial capacity gains are achievable in wireless communication systems employing a Multiple Input Multiple Output (MIMO) architecture using multiple antennas. Additionally, the employment of a MIMO architecture allows for the efficient exploitation of the spatial diversity available in a wireless MIMO environment, thus improving both the system's transmission integrity, as well as capacity.

The most potent space-time detection method found in the literature is constituted by the Sphere Decoder (SD) [2], which is capable of achieving the optimum performance of the Maximum Likelihood (ML) detector at a relatively low computational complexity. The SD was first proposed for employment in the context of space-time processing in [3]. The complex-valued version of the sphere decoder was proposed by Hochwald and ten Brink in [4]. The subject was

Manuscript received September 9, 2005; revised March 13, 2006; accepted July 10, 2006. The associate editor coordinating the review of this paper and approving it for publication was D. Huang. The work reported in this paper has formed part of the Wireless Enabling Techniques work area of the Core 3 Research Programme of the Virtual Centre of Excellence in Mobile and Personal Communications, Mobile VCE, www.mobilevce.com, whose funding support, including that of EPSRC, is gratefully acknowledged. Fully detailed technical reports on this research are available to Industrial Members of Mobile VCE.

The authors are with the School of ECS, Univ. of Southampton, SO17 1BJ, UK (e-mail: j.akhtman@soton.ac.uk; aw03r@ecs.soton.ac.uk; sqc@ecs.soton.ac.uk; lh@ecs.soton.ac.uk).

Digital Object Identifier 10.1109/TWC.2007.05710. further investigated by Damen et al. in [5]. Subsequently, an improved version of the Complex Sphere Decoder (CSD) was advocated by Pham et al. in [6].

Furthermore, CSD-aided detection was considered by Cui and Tellambura et al. in the context of a joint channel estimation and data detection scheme considered in [7], while a revised version of the CSD method, namely the so-called Multistage Sphere Decoding (MSD) was introduced in [8]. The generalized version of the sphere decoder, which is suitable for employment in rank-deficient MIMO systems supporting more transmitters than the number of receive antennas was introduced by Damen et al. in [9] and further refined by Cui and Tellambura in [10]. Additional variant of the sphere decoder algorithm with improved search radius was contributed by Zhao and Giannakis in [11].

Against this background, in this paper we propose a novel SDM detection method, which we refer to as the Soft-output OPtimized HIErarchy (SOPHIE) Spatial Division Multiplexing (SDM) detector. The proposed method may be regarded as an advanced extension of the SD methods portrayed in [6] and [10].

More specifically, our method can be employed in the above-mentioned rank-deficient and hence overloaded scenario, where the number of transmit antenna elements exceeds that of the receive antenna elements. Furthermore our scheme is suitable for high-throughput modulation schemes such as 16- and 64-QAM. We introduce a list of optimization rules, which facilitate the achievement of the near optimum BER performance of a Log-MAP detector at a relatively low computational complexity. The trade-off between the achievable BER performance and the associated computational complexity is controlled using two parameters. The proposed detection method exhibits two major advantages over all previously proposed techniques:

1) The bit-related soft information, which facilitates the achievement of near-optimum Log-MAP performance, is attained at the expense of a modest complexity increase over that of hard-decision ML detection.

2) Our method exhibits a particularly low polynomial complexity in both the low- and high-SNR regions.

In the critical range of SNR values, which corresponds to the "waterfall" region of the BER versus SNR curve, the detection complexity versus the number of transmit antennas remains exponential. Nevertheless, we demonstrate that the complexity can be dramatically reduced at the cost of a minor BER degradation. 
The rest of this paper is structured as follows. The OHRSAaided SDM detection methods considered are outlined in Section II. Specifically, in Section II-A we derive the OHRSAaided ML SDM detector, which benefits from the optimal performance of the ML SDM detector [12], while exhibiting a relatively low computational complexity. To elaborate a little further, in Section II-B we derive a bit-wise OHRSA-aided ML SDM detector, which allows us to apply the OHRSA method of Section II in high-throughput modulation schemes, such as $M$-QAM [12].

In Section II-C our discourse evolves further by deducing the OHRSA-aided Log-MAP SDM detector, which allows for an efficient evaluation of the soft-bit information and therefore results in highly efficient turbo decoding ${ }^{1}$. Unfortunately, however, in comparison to the OHRSA-aided ML SDM detector of Section II-B, the OHRSA-aided Log-MAP SDM detector of Section II-C exhibits a substantially higher complexity. Consequently, in Section II-D we derive an approximate Log-MAP method, namely the SOPHIE SDM detector. The SOPHIE SDM detector combines the advantages of both the OHRSA-aided ML and OHRSA-aided Log-MAP SDM detectors of Sections II-B and II-C, respectively. Specifically, it exhibits a similar performance to that of the optimal LogMAP detector, while imposing a relatively modest complexity. The computational complexity as well as the achievable performance of the SOPHIE SDM detector of Section II-D are quantified in Section III-B. Finally, our conclusions are offered in Section IV.

\section{Optimized Hierarchy Reduced SEARCH ALGORITHM (OHRSA) SDM DETECTION}

\section{A. OHRSA-Aided ML Detection}

We commence our discourse by deriving an OHRSAaided ML SDM detection method for a constant-modulus modulation scheme, such as $M$-PSK, where the transmitted symbols $s$ satisfy the condition of $|s|^{2}=1, \forall s \in \mathcal{M}$, and $\mathcal{M}$ denotes the set of $M$ complex-valued constellation points. In the next section, we will then demonstrate that the method derived is equally applicable for high-throughput multi-level modulation schemes, such as $M$-QAM.

Our system model is given by

$$
\mathbf{y}=\mathbf{H s}+\mathbf{w}
$$

where $\mathbf{y}, \mathbf{s}$ and $\mathbf{w}$ are the received signal, transmitted signal and Gaussian noise vectors, respectively, while $\mathbf{H}$ is $\left(n_{\mathrm{r}} \times m_{\mathrm{t}}\right)$ dimensional MIMO channel matrix. As outlined in [12] , the ML SDM detector provides an $m_{\mathrm{t}}$-antenna-based estimated signal vector candidate $\hat{\mathbf{s}}$, which maximizes the objective function defined as the conditional a posteriori probability function $\mathrm{P}\{\check{\mathbf{s}} \mid \mathbf{y}, \mathbf{H}\}$ over the set $\mathcal{M}^{m_{\mathrm{t}}}$ of legitimate solutions. More explicitly, we have

$$
\hat{\mathbf{s}}=\arg \max _{\check{\mathbf{s}} \in \mathcal{M}^{m_{t}}} P\{\check{\mathbf{s}} \mid \mathbf{y}, \mathbf{H}\},
$$

\footnotetext{
${ }^{1}$ Part of the material discussed in Sections II and II-C will also appear in [13], where we discuss the benefits of the soft-input-soft-output iterative MIMO detection.
}

where $\mathcal{M}^{m_{\mathrm{t}}}$ is the set of all possible $m_{\mathrm{t}}$-dimensional candidate symbol vectors of the $m_{\mathrm{t}}$-antenna-based transmitted signal vector $\mathbf{s}$. More specifically, we have

$$
\mathcal{M}^{m_{\mathrm{t}}}=\left\{\check{\mathbf{s}}=\left(\check{s}_{1}, \cdots, \check{s}_{m_{\mathrm{t}}}\right)^{\mathrm{T}} ; \check{s}_{i} \in \mathcal{M}\right\} .
$$

Furthermore, it was shown in [12] that we have

$$
\mathrm{P}\{\check{\mathbf{s}} \mid \mathbf{y}, \mathbf{H}\}=A \exp \left[-\frac{1}{\sigma_{w}^{2}}\|\mathbf{y}-\mathbf{H} \check{\mathbf{s}}\|^{2}\right],
$$

where $A$ is a constant, which is independent of any of the values $\left\{\check{s}_{i}\right\}_{i=1, \cdots, m_{\mathrm{t}}}$, while $\sigma_{w}^{2}$ denotes the Gaussian noise variance. Thus, it may be shown [12] that the probability maximization problem of Equation (2) is equivalent to the corresponding Euclidean distance minimization problem. Specifically, we have

$$
\hat{\mathbf{s}}=\arg \min _{\check{\mathbf{s}} \in \mathcal{M}^{m_{\mathrm{t}}}}\|\mathbf{y}-\mathbf{H} \check{\mathbf{s}}\|^{2},
$$

where the probability-based objective function of Equation (2) is substituted by the objective function determined by the Euclidean distance between the received signal vector $\mathbf{y}$ and the corresponding product of the channel matrix $\mathbf{H}$ with the a priori candidate of the transmitted signal vector $\check{\mathrm{s}} \in \mathcal{M}^{m_{\mathrm{t}}}$.

Consequently, our detection method relies on the observation, which was first considered in [10] and may be summarized in the following lemma.

Lemma 1: The ML solution of Equation (2) of a noisy linear problem described by Equation (1), where $s$ satisfies $|s|^{2}=1$, is given by

$$
\hat{\mathbf{s}}=\arg \min _{\breve{\mathbf{s}} \in \mathcal{M}^{m_{\mathrm{t}}}}\left\{\|\mathbf{U}(\check{\mathbf{s}}-\hat{\mathbf{x}})\|^{2}\right\},
$$

where $\mathbf{U}$ is an upper-triangular matrix having positive realvalued elements on the main diagonal and satisfying

$$
\mathbf{U}^{\mathrm{H}} \mathbf{U}=\left(\mathbf{H}^{\mathrm{H}} \mathbf{H}+\sigma_{w}^{2} \mathbf{I}\right)
$$

while

$$
\hat{\mathbf{x}}=\left(\mathbf{H}^{\mathrm{H}} \mathbf{H}+\sigma_{w}^{2} \mathbf{I}\right)^{-1} \mathbf{H}^{\mathrm{H}} \mathbf{y}
$$

is the unconstrained MMSE estimate of the transmitted signal vector $\mathbf{s}$, which was derived in [12] .

Note: Observe that Lemma 1 imposes no constraints on the dimensions, or rank of the matrix $\mathbf{H}$ of the linear system described by Equation (1). This property is particularly important, since it enables us to apply our proposed detection technique to the scenario of over-loaded systems, where the number of transmit antenna elements exceeds that of the receive antenna elements.

Proof of Lemma 1: It is evident that in contrast to the matrix $\mathbf{H}^{\mathrm{H}} \mathbf{H}$, the matrix $\left(\mathbf{H}^{\mathrm{H}} \mathbf{H}+\sigma_{w}^{2} \mathbf{I}\right)$ of Equation (6) is always Hermitian and positively definite, regardless of the rank of the channel matrix $\mathbf{H}$ associated with the particular MIMO channel realization encountered. Consequently, it may be represented as the product of an upper-triangular matrix $\mathbf{U}$ and its Hermitian adjoint matrix $\mathbf{U}^{\mathrm{H}}$ using for example the Cholesky factorization method [14]. 
Let $\mathbf{U}$ be the matrix generated by the Cholesky decomposition of the Hermitian positive definite matrix $\left(\mathbf{H}^{\mathrm{H}} \mathbf{H}+\sigma_{w}^{2} \mathbf{I}\right)$ of Equation (7). More specifically, we have

$$
\mathbf{U}^{\mathrm{H}} \mathbf{U}=\left(\mathbf{H}^{\mathrm{H}} \mathbf{H}+\sigma_{w}^{2} \mathbf{I}\right)
$$

where $\mathbf{U}$ is an upper-triangular matrix having positive realvalued elements on its main diagonal.

Upon expanding the objective function of Equation (6) and subsequently invoking Equation (7) we obtain

$$
\begin{aligned}
J(\check{\mathbf{s}}) & =\|\mathbf{U}(\check{\mathbf{s}}-\hat{\mathbf{x}})\|^{2} \\
& =(\check{\mathbf{s}}-\hat{\mathbf{x}})^{\mathrm{H}} \mathbf{U}^{\mathrm{H}} \mathbf{U}(\check{\mathbf{s}}-\hat{\mathbf{x}}) \\
& =(\check{\mathbf{s}}-\hat{\mathbf{x}})^{\mathrm{H}}\left(\mathbf{H}^{\mathrm{H}} \mathbf{H}+\sigma_{w}^{2} \mathbf{I}\right)(\check{\mathbf{s}}-\hat{\mathbf{x}}) \\
& =\check{\mathbf{s}}^{\mathrm{H}}\left(\mathbf{H}^{\mathrm{H}} \mathbf{H}+\sigma_{w}^{2} \mathbf{I}\right) \check{\mathbf{s}}-\hat{\mathbf{x}}^{\mathrm{H}}\left(\mathbf{H}^{\mathrm{H}} \mathbf{H}+\sigma_{w}^{2} \mathbf{I}\right) \check{\mathbf{s}} \\
& -\check{\mathbf{s}}^{\mathrm{H}}\left(\mathbf{H}^{\mathrm{H}} \mathbf{H}+\sigma_{w}^{2} \mathbf{I}\right) \hat{\mathbf{x}}+\hat{\mathbf{x}}^{\mathrm{H}}\left(\mathbf{H}^{\mathrm{H}} \mathbf{H}+\sigma_{w}^{2} \mathbf{I}\right) \hat{\mathbf{x}} .
\end{aligned}
$$

Furthermore, substituting Equation (8) into (10) yields

$$
\begin{aligned}
J(\check{\mathbf{s}}) & =\check{\mathbf{s}}^{\mathrm{H}} \mathbf{H}^{\mathrm{H}} \mathbf{H} \check{\mathbf{s}}-\mathbf{y}^{\mathrm{H}} \mathbf{H} \check{\mathbf{s}}-\check{\mathbf{s}}^{\mathrm{H}} \mathbf{H}^{\mathrm{H}} \mathbf{y} \\
& +\sigma_{w}^{2} \check{\mathbf{s}}^{\mathrm{H}} \check{\mathbf{s}}+\mathbf{y}^{\mathrm{H}} \mathbf{H}\left(\mathbf{H}^{\mathrm{H}} \mathbf{H}+\sigma_{w}^{2} \mathbf{I}\right)^{-1} \mathbf{H}^{\mathrm{H}} \mathbf{y} \\
& =\|\mathbf{y}-\mathbf{H} \check{\mathbf{s}}\|^{2}+\underbrace{\sigma_{w}^{2} \check{\mathbf{s}}^{\mathrm{H}} \check{\mathbf{s}}+\mathbf{y}^{\mathrm{H}}\left(\mathbf{H}\left(\mathbf{H}^{\mathrm{H}} \mathbf{H}+\sigma_{w}^{2} \mathbf{I}\right)^{-1} \mathbf{H}^{\mathrm{H}}-\mathbf{I}\right) \mathbf{y}}_{\psi} .
\end{aligned}
$$

Observe that in the case of a system employing a constantmodulus modulation scheme, such as $M$-PSK, where we have $\check{\mathbf{s}}^{\mathrm{H}} \check{\mathbf{S}}=1, \psi$ of Equation (11) constitutes a real-valued scalar and its value does not depend on the argument $\check{\text { s of }}$ the minimization problem formulated in Equation (6). Consequently, the minimization of the objective function $J(\check{\mathbf{s}})$ of Equation (11) can be reduced to the minimization of the term $\|\mathbf{y}-\mathbf{H} \check{\mathbf{s}}\|^{2}$, which renders it equivalent to the minimization problem of Equation (5). This completes the proof.

Using Lemma 1, in particular the fact that the matrix $\mathbf{U}$ is an upper-triangular matrix, the objective function $J(\check{\mathbf{s}})$ of Equation (11) may be reformulated as follows

$$
\begin{aligned}
J(\check{\mathbf{s}}) & =\|\mathbf{U}(\check{\mathbf{s}}-\hat{\mathbf{x}})\|^{2}=(\check{\mathbf{s}}-\hat{\mathbf{x}})^{\mathrm{H}} \mathbf{U}^{\mathrm{H}} \mathbf{U}(\check{\mathbf{s}}-\hat{\mathbf{x}}) \\
& =\sum_{i=1}^{m_{\mathrm{t}}}\left|\sum_{j=i}^{m_{\mathrm{t}}} u_{i j}\left(\check{s}_{j}-\hat{x}_{j}\right)\right|^{2}=\sum_{i=1}^{m_{\mathrm{t}}} \phi_{i}\left(\check{\mathbf{s}}_{i}\right),
\end{aligned}
$$

where $J(\check{\mathbf{s}})$ and $\phi_{i}\left(\check{\mathbf{s}}_{i}\right)$ are positive real-valued cost and subcost functions, respectively. Elaborating a little further we have

$$
\begin{aligned}
\phi_{i}\left(\check{\mathbf{s}}_{i}\right) & =\left|\sum_{j=i}^{m_{\mathrm{t}}} u_{i j}\left(\check{s}_{j}-\hat{x}_{j}\right)\right|^{2} \\
& =|u_{i i}\left(\check{s}_{i}-\hat{x}_{i}\right)+\underbrace{\sum_{j=i+1}^{m_{\mathrm{t}}} u_{i j}\left(\check{s}_{j}-\hat{x}_{j}\right)}_{a_{i}}|^{2} .
\end{aligned}
$$

Note that the term $a_{i}$ is a complex-valued scalar, which is independent of the specific symbol value $\check{s}_{i}$ of the $i$ th element of the a priori candidate signal vector $\check{\text { s. }}$.

Furthermore, let $J_{i}\left(\check{\mathbf{s}}_{i}\right)$ be a Cumulative Sub-Cost (CSC) function recursively defined as

$$
\begin{aligned}
J_{m_{\mathrm{t}}}\left(\check{s}_{m_{\mathrm{t}}}\right) & =\phi_{m_{\mathrm{t}}}\left(\check{s}_{m_{\mathrm{t}}}\right)=\left|u_{m_{\mathrm{t}} m_{\mathrm{t}}}\left(\check{s}_{m_{\mathrm{t}}}-\hat{x}_{m_{\mathrm{t}}}\right)\right|^{2} \\
J_{i}\left(\check{\mathbf{s}}_{i}\right) & =J_{i+1}\left(\check{\mathbf{s}}_{i+1}\right)+\phi_{i}\left(\check{\mathbf{s}}_{i}\right), \quad i=m_{\mathrm{t}}-1, \cdots, 1,
\end{aligned}
$$

where we define the candidate subvector as $\check{\mathbf{s}}_{i}=\left[\check{s}_{i}, \cdots, \check{s}_{m_{\mathrm{t}}}\right]$. Clearly, $J_{i}\left(\check{\mathbf{s}}_{i}\right)$ exhibits the following properties

$$
\begin{aligned}
J(\check{\mathbf{s}}) & =J_{1}\left(\check{\mathbf{s}}_{1}\right)>J_{2}\left(\check{\mathbf{s}}_{2}\right)>\cdots>J_{m_{\mathrm{t}}}\left(\check{s}_{m_{\mathrm{t}}}\right)>0 \\
J_{i}\left(\check{\mathbf{s}}_{i}\right) & =J_{i}\left(\left\{\check{s}_{j}\right\}, j=i, \cdots, m_{\mathrm{t}}\right)
\end{aligned}
$$

for all possible realizations of $\hat{\mathbf{x}} \in \mathbb{C}^{m_{\mathrm{t}}}$ and $\check{\mathrm{s}} \in \mathcal{M}^{m_{\mathrm{t}}}$, where the space $\mathbb{C}^{m_{\mathrm{t}}}$ contains all possible unconstrained MMSE estimates $\hat{\mathbf{x}}$ of the transmitted signal vector $\mathbf{s}$.

Equations (15a) and (15b) enable us to employ a highly efficient reduced search algorithm, which decreases the number of objective function evaluations of the minimization problem outlined in Equation (6) to a small fraction of the set $\mathcal{M}^{m_{\mathrm{t}}}$. This reduced-complexity search algorithm is outlined in the next section.

1) Search Strategy: Firstly, we commence the recursive search process with the evaluation of the CSC function value $J_{m_{\mathrm{t}}}\left(\check{s}_{m_{\mathrm{t}}}\right)$ of Equation (14a). Secondly, at each recursive step $i$ of the search algorithm proposed we stipulate a series of hypotheses concerning the value of the $M$-ary transmitted symbol $s_{i}$ associated with the $i$ th transmit antenna element and subsequently calculate the conditioned sub-cost function $J_{i}\left(\check{\mathbf{s}}_{i}\right)$ of Equation $(14 \mathrm{~b})$, where $\check{\mathbf{s}}_{i}=\left(\check{s}_{i}, \cdots, \check{s}_{m_{\mathrm{t}}}\right)^{\mathrm{T}}$ denotes the subvector of the $m_{\mathrm{t}}$-antenna-based candidate vector $\check{\mathrm{s}}$ comprising only the indices higher than or equal to $i$. Furthermore, for each tentatively assumed value of $\check{s}_{i}$ we execute a successive recursive search step $i-1$, which is conditioned on the hypotheses made in all preceding recursive steps $j=$ $i, \cdots, m_{\mathrm{t}}$. As substantiated by Equations (13) and (14b), the value of the CSC function $J_{i}\left(\check{\mathbf{s}}_{i}\right)$ is dependent only on the values of the elements $\left\{\check{s}_{j}\right\}_{j=i, \cdots, m_{\mathrm{t}}}$ of the a priori candidate signal vector $\check{\mathbf{s}}$, which are hypothesized from step $j=m_{\mathrm{t}}$ up to the present step $i$ of our recursive process. At each arrival at the step $i=1$ of the recursive process, a complete candidate vector $\check{\mathbf{s}}$ is hypothesized and the corresponding value of the cost function $J(\check{\mathbf{s}})$ formulated in Equation (12) is evaluated.

Observe that the recursive hierarchical search procedure described above may be employed to perform an exhaustive search through all possible values of the transmitted signal vector $\check{\mathbf{s}}$ and the resultant search process is guaranteed to arrive at the ML solution $\check{\mathrm{s}}_{\mathrm{ML}}$, which minimizes the value of the cost function $J(\check{\mathbf{s}})$ of Equation (12). Fortunately however, as opposed to other ML search schemes, the search process described above can be readily optimized, resulting in a dramatic reduction of the associated computational complexity. Specifically, the potential optimization complexity gain originates from the fact that most of the hierarchical search branches can be discarded at an early stage of the recursive search process. The corresponding optimization rules proposed may be outlined as follows.

Rule 1: We reorder the system model of Equation (1) as suggested in [15]. Specifically, we apply the best-first detection strategy outlined in [2], [5], [12], which implies that the transmitted signal vector components are detected in the decreasing order of the associated channel quality. As it was advocated in [12], the quality of the channel associated with the $i$ th element of the transmitted signal vector $\mathbf{s}$ is determined by the norm of the $i$ th column of the channel matrix $\mathbf{H}$. Consequently, for the sake of applying the bestfirst detection strategy, the columns of the channel matrix $\mathbf{H}$ 
are sorted in the increasing order of their norm. Thus, the resultant, column-reordered channel matrix $\mathbf{H}$ complies with the following criterion

$$
\left\|(\mathbf{H})_{1}\right\|^{2} \leq\left\|(\mathbf{H})_{2}\right\|^{2} \leq \cdots \leq\left\|(\mathbf{H})_{m_{\mathrm{t}}}\right\|^{2}
$$

where $(\mathbf{H})_{i}$ denotes the $i$ th column of the channel matrix $\mathbf{H}$. Note that the elements of the transmitted signal vector $\mathbf{s}$ are reordered correspondingly, but their original order has to be reinstated in the final stage of the detection process.

Rule 2: We employ the Schnorr-Euchner search strategy [16]. More specifically, at each recursive detection index $i=m_{\mathrm{t}}, \cdots, 1$, the potential candidate values $\left\{c_{m}\right\}_{m=1, \cdots, M} \in \mathcal{M}$ of the transmitted signal component $s_{i}$ are considered in the increasing order of the corresponding value of the sub-cost function $\phi_{i}\left(\check{\mathbf{s}}_{i}\right)=\phi_{i}\left(c_{m}, \check{\mathbf{s}}_{i+1}\right)$ of Equation (13), where we have

$$
\phi_{i}\left(c_{1}, \check{\mathbf{s}}_{i+1}\right)<\cdots<\phi_{i}\left(c_{m}, \check{\mathbf{s}}_{i+1}\right)<\cdots<\phi_{i}\left(c_{M}, \check{\mathbf{s}}_{i+1}\right),
$$

and according to Equation (13)

$$
\begin{aligned}
\phi_{i}\left(c_{m}, \check{\mathbf{s}}_{i+1}\right) & =\left|u_{i i}\left(c_{m}-\hat{x}_{i}\right)+a_{i}\right|^{2} \\
& =u_{i i}\left|c_{m}-\hat{x}_{i}+\frac{a_{i}}{u_{i i}^{2}}\right|^{2} .
\end{aligned}
$$

Consequently, the more likely candidates $c_{m}$ of the $i$ th element of the transmitted signal vector $\mathbf{s}$ are examined first. Observe that the sorting criterion of Equation (17) may also be interpreted as a biased Euclidean distance of the candidate constellation point $c_{m}$ from the unconstrained MMSE estimate $\hat{x}_{i}$ of the transmitted signal component $s_{i}$.

Rule 3: We define a cut-off value of the cost function $J_{\min }=\min \{J(\check{\mathbf{s}})\}$ as the minimum value of the total cost function obtained up to the present point of the search process. Consequently, at each arrival at step $i=1$ of the recursive search process, the cut-off value of the cost function is updated as follows

$$
J_{\min }=\min \left\{J_{\min }, J(\check{\mathbf{s}})\right\} .
$$

Rule 4: Finally, at each recursive detection step $i$, only the high probability search branches corresponding to the highly likely symbol candidates $c_{m}$ resulting in low values of the CSC function obeying $J_{i}\left(c_{m}\right)<J_{\min }$ are pursued. Furthermore, as follows from the sorting criterion of the optimization Rule 2, as soon as the inequality $J_{i}\left(c_{m}\right)>J_{\min }$ is encountered, the search loop at the $i$ th detection step is discontinued.

Given the cost-functions of Equation (12) and the appropriately ordered matrix $\mathbf{H}$ of Equation (1), the proposed algorithm may be viewed as a specific manifestation of a tree search algorithm [17]. Another example of a tree search algorithm commonly employed in the design of communication systems constitutes the well-known Viterbi algorithm [18], [19]. More specifically, the sub-cost function values of Equation (13) may be regarded as being analogous to the the branch metrics, while the CSC values of Equation (14) as accumulated path metrics. It should be noted however, that the OHRSA-ML algorithm described here and the classic treesearch-based Viterbi algorithm exhibit substantial differences. Specifically, the Viterbi algorithm assumes that the branch
TABLE I

EXAMPLES OF QUANTIZATION VECTORS

\begin{tabular}{|c|l|}
\hline Modulation scheme & $\boldsymbol{q}^{\mathrm{T}}$ \\
\hline \hline BPSK & {$[1]$} \\
\hline QPSK & $\frac{1}{\sqrt{2}}[1, \jmath]$ \\
\hline 16QAM & $\frac{1}{\sqrt{10}}[1,1 \jmath, 2,2 \jmath]$ \\
\hline 64QAM & $\frac{1}{\sqrt{42}}[1,1 \jmath, 2,2 \jmath, 4,4 \jmath]$ \\
\hline
\end{tabular}

metric is a function of the system states constituting one particular state transition, which is equivalent to the assumption of having a diagonal matrix $\mathbf{U}$ in Equation (7). Evidently, this requirement cannot be satisfied by our generic MIMO system. Consequently, the tree-search-based Viterbi algorithm cannot be applied to the search problem described above.

\section{B. Bitwise OHRSA ML Detection}

In this section we generalize the result obtained in Section II-A to the case of systems employing high-throughput modulation schemes, namely $M$-QAM, where each modulated symbol belongs to a discrete phasor constellation $\mathcal{M}=$ $\left\{c_{m}\right\}_{m=1, \cdots, M}$. It is evident that each phasor point $c_{m}$ of an $M$-QAM constellation map may be represented as the inner product of a unique bit-based vector $\boldsymbol{d}_{m}=\left\{d_{m l}=\right.$ $-1,1\}_{l=1, \cdots, b}$ and the corresponding quantisation vector $\boldsymbol{q}$. Specifically, we have

$$
c_{m}=\boldsymbol{q}^{\mathrm{T}} \boldsymbol{d}_{m} .
$$

Some examples of the quantization vectors corresponding to the modulation schemes of QPSK, 16-QAM and 64-QAM are portrayed in Table I.

Furthermore, we define a $\left(b m_{\mathrm{t}} \times m_{\mathrm{t}}\right)$-dimensional quantization matrix $\mathbf{Q}=\mathbf{I} \otimes \boldsymbol{q}$, where $\mathbf{I}$ is an $\left(m_{\mathrm{t}} \times m_{\mathrm{t}}\right)$ dimensional identity matrix and $\boldsymbol{q}$ is the aforementioned quantization vector, while $\otimes$ denotes the matrix direct product [20]. Consequently the $M$-QAM-modulated signal vector $\mathbf{s}$ may be represented as

$$
\mathbf{s}=\mathbf{Q t}
$$

where $\mathbf{t}=\left[\boldsymbol{t}_{1}^{\mathrm{T}}, \cdots, \boldsymbol{t}_{m_{\mathrm{t}}}^{\mathrm{T}}\right]^{\mathrm{T}}$ is a column supervector comprising the bit-based vectors $\boldsymbol{t}_{i}$ associated with each transmitted signal vector component $s_{i}$. Substituting Equation (21) into the system model of Equation (1) yields

$$
\mathbf{y}=\mathbf{H Q t}+\mathbf{w} .
$$

Moreover, since $\mathbf{t}$ is a real-valued vector, we can elaborate a bit further and deduce a real-valued system model as follows $\tilde{\mathbf{y}}=\left[\begin{array}{c}\mathcal{R}\{\mathbf{y}\} \\ \mathcal{I}\{\mathbf{y}\}\end{array}\right]=\left[\begin{array}{c}\mathcal{R}\{\mathbf{H Q}\} \\ \mathcal{I}\{\mathbf{H Q}\}\end{array}\right] \mathbf{t}+\left[\begin{array}{c}\mathcal{R}\{\mathbf{w}\} \\ \mathcal{I}\{\mathbf{w}\}\end{array}\right]=\tilde{\mathbf{H}} \mathbf{t}+\tilde{\mathbf{w}}$,

where $\tilde{\mathbf{H}}$ is a real-valued $\left(2 n_{\mathrm{r}} \times b m_{\mathrm{t}}\right)$-dimensional bitwise channel matrix. Observe in Equation (22) that the requirement of having constant-modulus symbols is satisfied by the modified system model of Equation (22), since we have $\left|t_{i}\right|^{2}=1$ and thus the method described in Section II-A is applicable for the evaluation of the bitwise ML estimate $\hat{\mathbf{t}}$ of Equation (22). The resultant Bitwise OHRSA-aided ML SDM detector is summarized in Algorithm 1. 


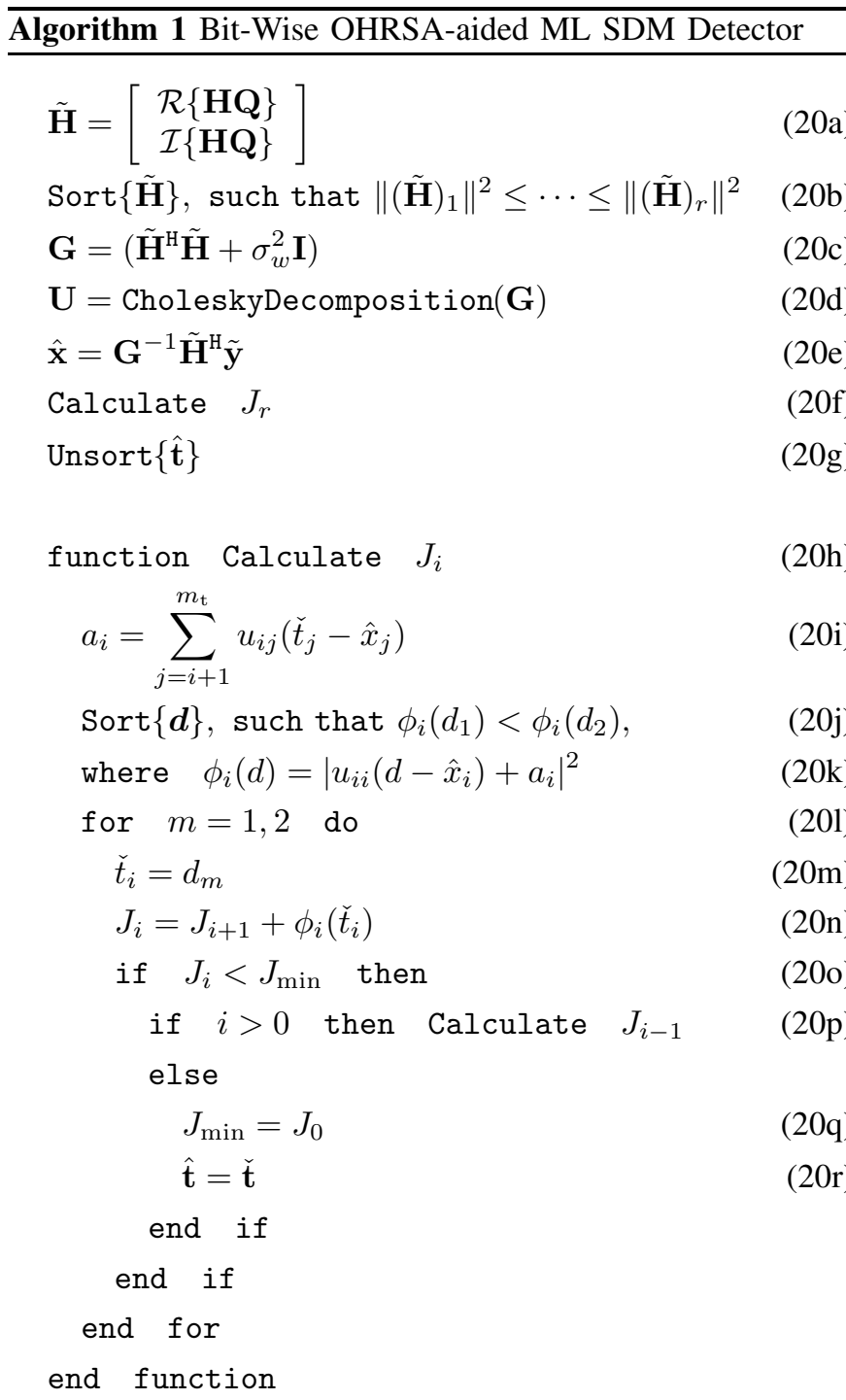

\section{OHRSA-Aided Log-MAP Detection}

Subsequently, the derivation of an expression for the evaluation of the soft-bit information associated with the bit estimates of the bitwise ML detector of Section II-B is given in [12]. More specifically, the soft-bit value associated with the $i$ th bit of estimated signal vector $\hat{\mathbf{t}}$ is determined by the log-likelihood ratio (LLR) function defined in [21] as

$$
L_{i}=\log \frac{\sum_{\check{\mathbf{t}} \in \mathcal{M}_{i}^{1 ; r}} \mathrm{P}\{\tilde{\mathbf{y}} \mid \check{\mathbf{t}}, \tilde{\mathbf{H}}\}}{\sum_{\check{\mathbf{t}} \in \mathcal{M}_{i}^{-1 ; r}} \mathrm{P}\{\tilde{\mathbf{y}} \mid \check{\mathbf{t}}, \tilde{\mathbf{H}}\}},
$$

where we define the constrained subset $\mathcal{M}_{i}^{d ; r}$ of the transmitted bitwise signal candidates $\check{\mathbf{t}}$ as follows

$\mathcal{M}_{i}^{d ; r}=\left\{\check{\mathbf{t}}=\left(\check{t}_{1}, \cdots, \check{t}_{r}\right)^{\mathrm{T}} ; \check{t}_{j} \in\{-1,1\}\right.$ for $\left.j \neq i, \check{t}_{i}=d\right\}$.

The direct calculation of the accumulate a posteriori conditional probabilities in the nominator and denominator of Equation (24) may have an excessive complexity in practice.
Fortunately, as advocated in [12], the expression in Equation (24) can be closely approximated as follows

$$
L_{i} \approx \log \frac{\mathrm{P}\left\{\tilde{\mathbf{y}} \mid \hat{\mathbf{t}}_{i}^{1}, \tilde{\mathbf{H}}\right\}}{\mathrm{P}\left\{\tilde{\mathbf{y}} \mid \hat{\mathbf{t}}_{i}^{-1}, \tilde{\mathbf{H}}\right\}},
$$

where we define the constrained ML estimate

$$
\hat{\mathbf{t}}_{i}^{d}=\arg \max _{\tilde{\mathbf{t}} \in \mathcal{M}_{i}^{d ; r}} \mathrm{P}\{\tilde{\mathbf{y}} \mid \check{\mathbf{t}}, \tilde{\mathbf{H}}\}, \quad d=-1,1 .
$$

As suggested by the nature of Equation (26), the detection process employing the objective function determined by Equations (26) and (27) is usually referred to as the Logarithmic Maximum A Posteriori (Log-MAP) probability detector.

A practical version of the Log-MAP detector may be derived as follows. Substituting Equation (4) into (26) yields

$$
L_{i} \approx \frac{1}{\sigma_{w}^{2}}\left[\left\|\tilde{\mathbf{y}}-\tilde{\mathbf{H}} \hat{\mathbf{t}}_{i}^{-1}\right\|^{2}-\left\|\tilde{\mathbf{y}}-\tilde{\mathbf{H}} \hat{\mathbf{t}}_{i}^{1}\right\|^{2}\right],
$$

where we have

$$
\hat{\mathbf{t}}_{i}^{d}=\arg \min _{\check{\mathbf{t}} \in \mathcal{M}_{i}^{d ; r}}\|\tilde{\mathbf{y}}-\tilde{\mathbf{H}} \check{\mathbf{t}}\|^{2}, \quad d=-1,1,
$$

and again, $\mathcal{M}_{i}^{d ; r}$ denotes the specific subset of the entire set $\mathcal{M}^{r}$ of bitwise signal vector candidates, which comprises the bit value $d=\{-1,1\}$ at the $i$ th bit position.

Furthermore, substituting the bitwise objective function of Equation (11) into (28) yields

$$
L_{i} \approx \frac{1}{\sigma_{w}^{2}}\left[J\left(\hat{\mathbf{t}}_{i}^{-1}\right)-J\left(\hat{\mathbf{t}}_{i}^{1}\right)\right],
$$

where $\hat{\mathbf{t}}_{i}^{d}$ and the corresponding cost function value $J\left(\hat{\mathbf{t}}_{i}^{d}\right)$ may be obtained by applying the constrained OHRSA-aided ML detection method of Algorithm 1.

Consequently, the evaluation of the bitwise Log-MAP estimates of the transmitted bitwise signal vector $\mathbf{t}$ involves repetitive evaluation of $2 r$ constrained ML estimates $\hat{\mathbf{t}}_{i}^{b}$ along with the associated $2 r$ values of the objective function $J\left(\hat{\mathbf{t}}_{i}^{b}\right)$.

\section{Soft-Output OHRSA-Aided Approximate Log-MAP Detec- tion}

Let us define the $(r \times 2)$-dimensional Bitwise Minimum Cost (BMC) function matrix $\hat{\mathbf{J}}$ having elements as follows

$$
\hat{J}_{i d}=J\left(\hat{\mathbf{t}}_{i}^{d}\right), i=1, \cdots, r, d=-1,1,
$$

where $\hat{\mathbf{t}}_{i}^{d}$ is defined by Equation (29). Using the BMC matrix of Equation (31), Equation (30) may also be expressed in a vectorial form as

$$
\mathbf{L}=\frac{1}{\sigma_{w}^{2}}\left[(\hat{\mathbf{J}})_{1}-(\hat{\mathbf{J}})_{2}\right]
$$

where, as before, $(\hat{\mathbf{J}})_{b}$ denotes the $d$ th column of the matrix $\hat{\mathbf{J}}$ having elements defined by Equation (31).

Consequently, in order to evaluate the bit-related soft information we have to populate the BMC matrix $\hat{\mathbf{J}}$ of Equation (31) with the corresponding values of the cost function of Equation (31). Observe, that the evaluation of the ML estimate $\hat{\mathbf{t}}$ will situate half elements of the cost matrix $\hat{\mathbf{J}}$ with the 


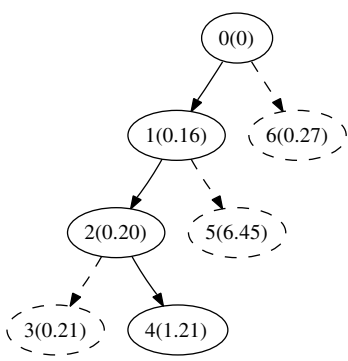

(a)

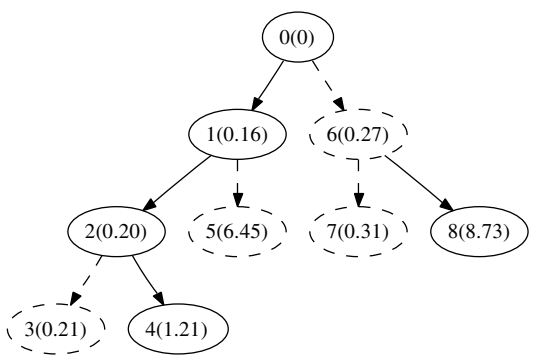

(b)

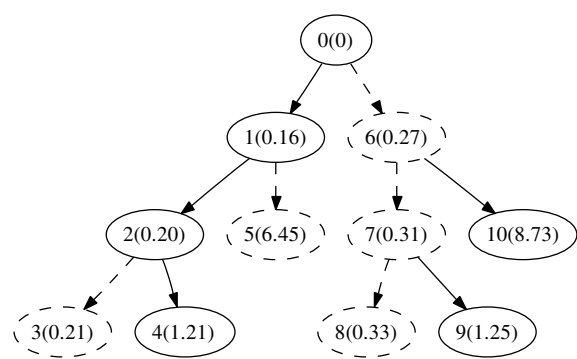

(c)

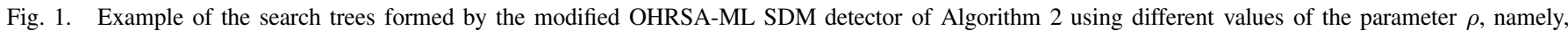

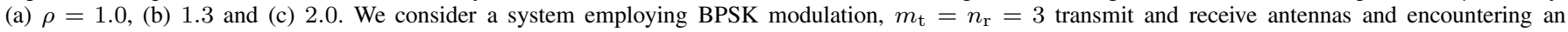

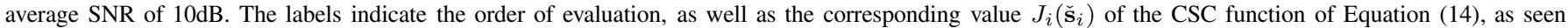
in the brackets.

corresponding minimum value of the cost function associated with the ML estimate, such that we have

$$
J_{i b}=J(\hat{\mathbf{t}}), i=1, \cdots, r, b=\hat{t}_{i} .
$$

Subsequently, let us introduce the following adjustments to Algorithm 1. Firstly, we introduce an additional parameter $\rho$, which we refer to as the search radius factor. More specifically, the parameter $\rho$ allows us to control the rate of convergence for the tree search process of Algorithm 1 and affects the cut-off value of a CSC function, which limits the passage of the recursive search process through low-likelihood search branches having a CSC function value $J_{i}\left(\check{\mathbf{t}}_{i}\right)$ in excess of $\rho J_{\min }$, as opposed to $J_{\min }$. Thus, the following rule replaces Rule 4 of Section II-A.1.

Rule 4a: At each recursive detection level $i$, only the high-probability search branches corresponding to the highly likely symbol candidates $d_{m}$ resulting in low values of the CSC function obeying $J_{i}\left(d_{m}\right)<\rho J_{\min }$ are pursued. Furthermore, as follows from the sorting criterion of the optimization Rule 2 , as soon as the inequality $J_{i}\left(d_{m}\right)>\rho J_{\min }$ is satisfied, the search loop at the $i$ th recursive detection level is discontinued.

Secondly, we introduce an additional rule, which facilitates the evaluation of the elements of the BMC matrix $\hat{\mathbf{J}}$ of Equation (31). Explicitly, we postulate Rule 5.

Rule 5: At each arrival at the bottom of the search tree, which corresponds to search level 1 , the resultant value of the branch cost function $J(\check{\mathbf{t}})$ is utilized to populate the elements of the BMC matrix $\hat{\mathbf{J}}$, which correspond to the bitwise signal components $\check{t}_{i}$ comprising the obtained signal candidate $\check{\mathbf{t}}$. Namely, we have

$$
\hat{J}_{i d}=\min \left\{\hat{J}_{i d}, J(\check{\mathbf{t}})\right\}, i=1, \cdots, r, d=\check{t}_{i} .
$$

Subsequently, we suggest that the evaluation of the BMC matrix $\hat{\mathbf{J}}$, which is performed in the process of the ML search of Algorithm 1 extended by Rule 4a and using Rule 5 will allow us to provide reliable soft-bit information, while imposing a relatively low computational complexity. The main rationale of this assumption will be outlined in our quantitative complexity and performance analysis portrayed in Section IIIB.

As we will further demonstrate in Section III-B, the resultant approximate Log-MAP SDM detector exhibits a particularly low complexity at high SNR values. On the other hand, at low SNR values the associated complexity substantially increases. Consequently, in order to control the computational complexity at low SNR values, we introduce an additional complexity-control parameter, namely the search resolution parameter $\gamma$. Our aim is to avoid the computationally demanding and yet inefficient detection of the specific signal components, which have their signal energy well below the noise floor. More specifically, we modify Equation (20p) of Algorithm 1 according to Rule 6.

Rule 6: The branching of the tree search described by Algorithm 1 is truncated, if the SNR associated with the corresponding signal component is lower than the value of the search resolution parameter $\gamma$. In other words, the search along a given branch is truncated if we have $\frac{\left\|\tilde{\mathbf{H}}_{i}\right\|^{2}}{\sigma_{w}^{2}}<\gamma$.

Upon applying Rules 4, 5 and 6 in the context of the OHRSA-ML method of Algorithm 1, we arrive at an approximate OHRSA-Log-MAP SDM detector, which avoids the repetitive search required by the OHRSA-Log-MAP SDM detector of Section II-C.

The search tree diagram generated by applying the approximate OHRSA-Log-MAP SDM detector described above in the scenario of using BPSK and employing $m_{\mathrm{t}}=n_{\mathrm{r}}=3$ transmit and receive antennas as well as assuming $\rho=1.0,1.3$ and $\rho=2.0$ are depicted in Figs. 1 (a), (b) and (c), respectively. Observe that the higher the value of $\rho$, the slower the convergence of the search process is. The suitable value of the search radius factor $\rho$ may be found empirically and its exact value within a sensible range has in fact little effect on the attainable performance and the associated complexity of the proposed algorithm. The impact of the choice of the particular value of the parameter $\rho$ on both the BER performance and computational complexity is further analysed in Section III-B.

The resultant OHRSA-aided approximate Log-MAP SDM detector, which we refer to as the Soft-output OPtimized HIErarchy (SOPHIE) SDM detector is summarized in Algorithm 2.

\section{QUANTITATIVE ANALYSIS}

\section{A. SDM-OFDM System Model}

In order to illustrate a practical application example of the detection method proposed, let us consider an SDM-OFDM wireless communication system employing $m_{\mathrm{t}}$ transmit and $n_{\mathrm{r}}$ receive antennas and operating over a wideband multi-path Rayleigh channel [12]. 


\section{$\overline{\text { Algorithm } 2 \text { SOPHIE Approximate Log-MAP SDM Detector }}$}

$$
\begin{aligned}
& \tilde{\mathbf{H}}=\left[\begin{array}{c}
\mathcal{R}\{\mathbf{H Q}\} \\
\mathcal{I}\{\mathbf{H Q}\}
\end{array}\right] \\
& \text { Sort }\{\tilde{\mathbf{H}}\}, \text { such that }\left\|(\tilde{\mathbf{H}})_{1}\right\|^{2} \leq \cdots \leq\left\|(\tilde{\mathbf{H}})_{r}\right\|^{2} \\
& \mathbf{G}=\left(\tilde{\mathbf{H}}^{\mathrm{H}} \tilde{\mathbf{H}}+\sigma_{w}^{2} \mathbf{I}\right) \\
& \mathbf{U}=\text { CholeskyDecomposition }(\mathbf{G}) \\
& \hat{\mathbf{x}}=\mathbf{G}^{-1} \tilde{\mathbf{H}}^{\mathrm{H}} \tilde{\mathbf{y}} \\
& \text { Calculate } \quad J_{r} \\
& \mathbf{L}=\frac{1}{\sigma_{w}^{2}}\left[(\hat{\mathbf{J}})_{0}-(\hat{\mathbf{J}})_{1}\right] \\
& \text { Unsort }\left\{L_{i}\right\}_{i=1, \cdots, r}
\end{aligned}
$$

$$
\begin{aligned}
& \text { function Calculate } J_{i} \\
& a_{i}=\sum_{j=i+1}^{m_{\mathrm{t}}} u_{i j}\left(\check{t}_{j}-\hat{x}_{j}\right) \\
& \text { Sort }\{\boldsymbol{d}\} \text {, such that } \phi_{i}\left(d_{1}\right)<\phi_{i}\left(d_{2}\right) \text {, } \\
& \text { where } \phi_{i}(d)=\left|u_{i i}\left(d-\hat{x}_{i}\right)+a_{i}\right|^{2} \\
& \text { for } m=1,2 \text { do } \\
& \check{t}_{i}=d_{m} \\
& J_{i}=J_{i+1}+\phi_{i}\left(\check{t}_{i}\right) \\
& \text { if } J_{i}<\rho J_{\min } \text { then } \\
& \text { if } i>0 \text { and } \frac{\left\|(\tilde{\mathbf{H}})_{i}\right\|^{2}}{\sigma_{w}^{2}}>\gamma \text { then } \\
& \text { Calculate } J_{i-1} \\
& \text { else } \\
& J_{\min }=\min \left(J_{i}, J_{\min }\right) \\
& \text { for } j=1, \cdots, r \\
& \hat{J}_{j \breve{t}_{j}}=\min \left\{\hat{J}_{j \check{t}_{j}}, J(\check{\mathbf{t}})\right\}, j=1, \cdots, r \\
& \text { end for } \\
& \text { end if } \\
& \text { end if } \\
& \text { end for }
\end{aligned}
$$$$
\text { end function }
$$

The orthogonal multi-carrier structure of our SDM-OFDM transceiver allows us to characterise the broadband frequencyselective channel considered as an OFDM subcarrier-related vector of flat-fading Channel Transfer Function (CTF) coefficients. For each OFDM symbol $n$ and subcarrier $k$ the MIMO channel is characterized by a $\left(n_{\mathrm{r}} \times m_{\mathrm{t}}\right)$-dimensional matrix $\mathbf{H}[n, k]$ of the CTF coefficients associated with the different propagation links, such that the element $H_{i j}[n, k]$ of the CTF matrix $\mathbf{H}[n, k]$ corresponds to the propagation link connecting the $j$ th transmit and $i$ th receive antennas [12].

Consequently, our simulations were performed in the baseband frequency domain. We assume having a total bandwidth of $800 \mathrm{kHz}$. The OFDM system utilises 128 orthogonal subcarriers. For forward error correction (FEC) we use $\frac{1}{2}$-rate turbo coding [19] employing two constraint-length $K=3$ Recursive Systematic Convolutional (RSC) component codes [22]. The octally represented RCS generator polynomials of $(7,5)$ were used. The transmitted information bits are interleaved over 8 subsequent OFDM symbols. Furthermore, we employ the eight-path urban non-line-of-sight Bug Rayleighfading channel model characterised in [23]. We assumed a block-fading channel, having an OFDM-symbol-normalized Doppler frequency of 0.1 . Finally, throughout this section we stipulate the assumption of perfect channel knowledge, where the knowledge of the frequency-domain subcarrierrelated coefficients $H[n, k]$ is deemed to be available in the receiver.

\section{B. Complexity and Performance Analysis}

The direct calculation of the complexity associated with the SOPHIE of Algorithm 2 is somewhat challenging, since the complexity is a random variable, which is a function of several parameters, such as the numbers $m_{\mathrm{t}}$ and $n_{\mathrm{r}}$ of the transmit and receive antennas, the average SNR encountered as well as the values of the complexity-control parameters $\rho$ and $\gamma$ in Algorithm 2. Therefore, we statistically characterize the corresponding complexity using computer simulations.

Fig. 2 characterises the achievable performance as well as the associated computational complexity exhibited by the $4 \times 4$ 16QAM-SDM-OFDM system employing the SOPHIE SDM detector of Algorithm 2. More specifically, we analyse the associated performance versus complexity trade-offs of using various values of the search radius factor and search resolution parameters $\rho$ and $\gamma$, respectively. In Fig. 2(a) we can observe how the achievable BER performance (top) and the corresponding computational complexity depend on the value of the parameter $\gamma$. Using the results depicted in Fig. 2(a) we may conclude that the optimum choice of the search resolution parameter $\gamma$ lies in the range of $0.5-0.8$, where we have a minor BER versus SNR performance degradation of less than $0.5 \mathrm{~dB}$, while achieving up to two orders of magnitude complexity reduction at low SNR values, when compared to the full-complexity SOPHIE algorithm assuming $\gamma=0$.

On the other hand, Fig. 2(b) portrays both the achievable BER performance and the associated complexity of the $4 \times$ 4 16QAM-SDM-OFDM system for different values of the search radius factor parameter $\rho$. We may conclude that the optimum trade-off between the attainable BER performance and the associated complexity is achieved, when the value of the search radius factor parameter $\rho$ lies in the range of $1.3-1.5$, where the BER performance degradation imposed does not exceed $0.5 \mathrm{~dB}$, while the associated computational complexity is reduced by more than an order of magnitude, when compared to large values of $\rho$, such as for instance $\rho=$ 2.0 .

Furthermore, Fig. 4(a) demonstrates both the BER performance (top) and the associated computational complexity exhibited by the $(8 \times 8) \mathbf{4 , 1 6}$ and $\mathbf{6 4 Q A M}$ SDM-OFDM systems employing the SOPHIE SDM detector of Algorithm 2 and assuming $\rho=1.3, \gamma=0.8$.

Fig. 4(b) characterises the 16QAM-SDM-OFDM system employing the SOPHIE SDM detector of Algorithm 2 and 

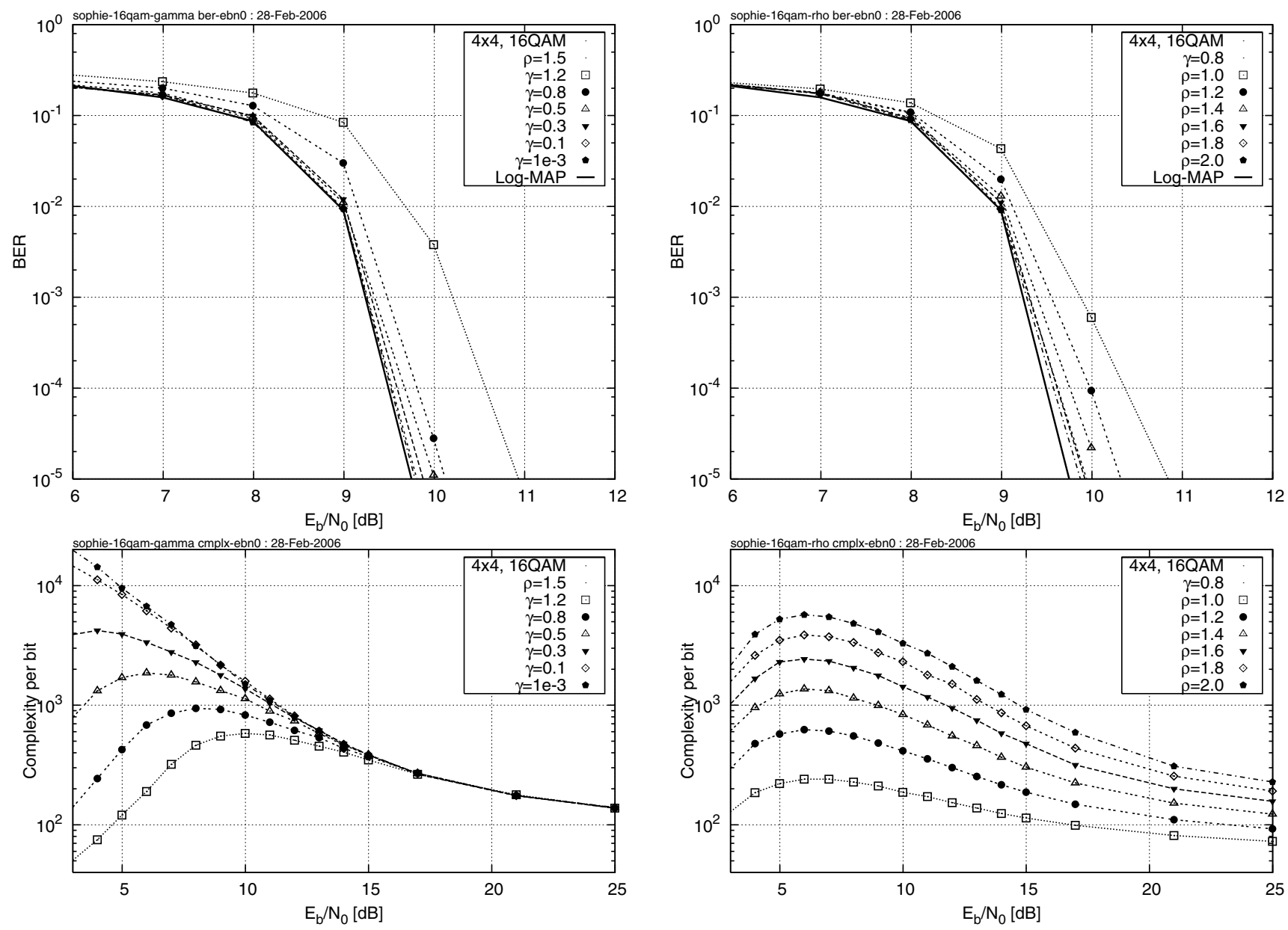

(a)

(b)

Fig. 2. Bit Error Rate (top) and the associated computational complexity per detected bit (botom) exhibited by the $4 \times 4$ 16QAM-SDM-OFDM system employing the SOPHIE SDM detector of Algorithm 2 and assuming different values of the search resolution and search radius parameters (a) $\gamma$ and (b) $\rho$, respectively. The abscissa represents the average $E_{b} / N_{0}$ recorded at the receive antenna elements.

having a constant number of $n_{\mathrm{r}}=4$ receive antenna elements in terms of its ability to detect the multiplexed signals arriving from various numbers of transmit antenna elements. Specifically, we aim for exploring the performance of the SOPHIE SDM detector in the overloaded system scenario, where the number of transmit antenna elements exceeds that of the receiver elements and thus we have $m_{\mathrm{t}}>n_{\mathrm{r}}$.

Indeed, the BER curves portrayed in Fig. 4 (top) confirm the near-Log-MAP performance of the SOPHIE SDM detector of Algorithm 2 in both systems employing high-throughput modulation schemes as well as in the rank-deficient overloaded system scenario.

Finally, Fig. 3 characterizes the computational complexity imposed by the SOPHIE SDM detector of Algorithm 2 as a function of the number $m_{\mathrm{t}}=n_{\mathrm{r}}$ of transmit and receive antennas. More specifically, we consider three ranges of SNR values: low SNRs, the critical SNR, which corresponds to the "waterfall" region of the BER versus SNR curve, as well as high SNRs, which corresponds to the error-free detection region. In Fig. 3 we may observe that the computational complexity imposed by the SOPHIE detector increases according to a polynomial law as a function of the number of transmit antennas for both high and low SNRs.

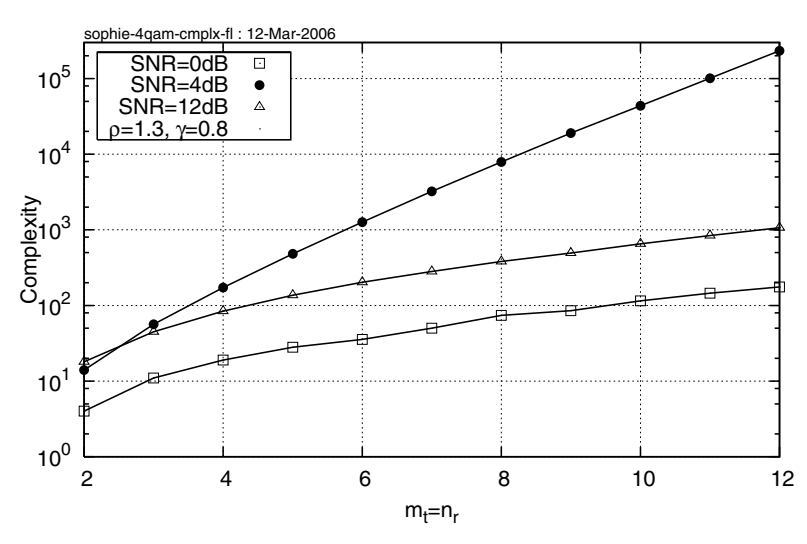

Fig. 3. Computational Complexity, imposed by the MIMO-OFDM system employing the SOPHIE SDM detector of Algorithm 2 and assuming $\rho=1.3$, $\gamma=0.8$. The complexity is quantified in terms of total number of real additions and multiplications as a function of the number $m_{\mathrm{t}}=n_{\mathrm{r}}$ of transmit and receive antennas.

\section{CONCLUSION}

In this paper we proposed a so-called SOPHIE SDM detection method, which may be regarded as an advanced extension of the CSD method. We have shown that the SOPHIE SDM 
detector proposed closely approaches the optimum performance of the Log-MAP detector while imposing a dramatically lower computational complexity. Our method is readily applicable to OFDM systems employing high-throughput modulation schemes, such as $M$-QAM. Additionally, the SOPHIE detector may be employed in the over-loaded scenario, where the number of transmit antenna elements exceeds that of the receive antenna elements, while the associated computational complexity increases only moderately even in heavily overloaded scenarios and is almost independent of the number of receive antennas. The method proposed maintains the nearoptimal performance of the Log-MAP SDM detector even in the heavily over-loaded scenarios.

\section{REFERENCES}

[1] A. Goldsmith, S. A. Jafar, N. Jindal, and S. Vishwanath, "Capacity limits of MIMO channels," IEEE J. Select. Areas Commun., vol. 21, no. 5, pp. 684-702, June 2003.

[2] U. Fincke and M. Pohst, "Improved method for calculating vector of short length in a lattice, including a complexity analysis," Math. Comput., vol. 44, pp. 463-471, Apr. 1985

[3] M. O. Damen, A. Chkeif, and J.-C. Belfiore, "Lattice code decoder for space-time codes," IEEE Commun. Lett., pp. 161-163, May 2000.

[4] B. Hochwald and S. ten Brink, "Achieving near-capacity on a multipleantenna channel," IEEE Trans. Commun., vol. 51, no. 3, pp. 389-399, Mar. 2003.

[5] M. O. Damen, H. E. Gamal, and G. Caier, "On maximum-likelihood detection and the search for closest lattice point," IEEE Trans. Inform. Theory, vol. 49, no. 10, pp. 2389-2402, Oct. 2003.

[6] D. Pham, K. R. Pattipati, P. K. Willet, and J. Luo, "An improved complex sphere decoder for V-BLAST systems," IEEE Signal Processing Lett., vol. 11, no. 9, pp. 748-751, Sep. 2004.

[7] T. Cui and C. Tellambura, "Joint channel estimation and data detection for OFDM systems via sphere decoding," in Proc. IEEE Global Telecommun. Conf., Nov.-Dec. 2004, vol. 6, pp. 3656-3660.

[8] _ - "Approximate ML detection for MIMO systems using multistage sphere decoding," IEEE Signal Processing Lett., vol. 12, no. 3, pp. 222225, Mar. 2005.

[9] M. Damen, K. Abed-Meraim, and J.-C. Belfiore, "Generalised sphere decoder for asymmetrical space-time communication architecture," IEEE Electron. Lett., vol. 36, no. 2, pp. 166-167, Jan. 2000.

[10] T. Cui and C. Tellambura, "An efficient generalized sphere decoder for rank-deficient MIMO systems," IEEE Commun. Lett., vol. 9, no. 5, pp. 423-425, May 2005.

[11] W. Zhao and G. Giannakis, "Sphere decoding algorithms with improved radius search," IEEE Trans. Commun., vol. 53, no. 7, pp. 1104-1109, July 2005.

[12] L. Hanzo, M. Münster, B. J. Choi, and T. Keller, OFDM and MC-CDMA for Broadband Multi-User Communications, WLANs and Broadcasting. New York: John Wiley and IEEE Press, 2003.

[13] A. Wolfgang, J. Akhtman, S. Chen, and L. Hanzo, "Iterative MIMO detection for rank-deficient systems," IEEE Signal Processing Lett., vol. 13 , no. 11 , pp. 699-702, Nov. 2006

[14] J. E. Gentle, Numerical Linear Algebra for Applications in Statistics. Berlin: Springer-Verlag, 1998.

[15] M. K. Varanasi, "Decision feedback multiuser detection: A systematic approach," IEEE Trans. Inform. Theory, vol. 45, pp. 219-240, Jan. 1999.

[16] C.-P. Schnorr and M. Euchner, "Lattice basis reduction: Improved practical algorithms and solving subset sum problems." In Fundamentals of Computation Theory, pp. 68-85, 1991. [Online.] Available: citeseer.ist.psu.edu/schnorr93lattice.html
[17] G. Valiente, Algorithms on Trees and Graphs. New York: Springer, 2002.

[18] A. Viterbi, CDMA: Principles of Spread Spectrum Communication. Reading MA: Addison-Wesley, 1995.

[19] L. Hanzo, T. H. Liew, and B. L. Yeap, Turbo Coding, Turbo Equalisation and Space-Time Coding. Chichester, UK; Piscataway, NJ, USA: John Wiley and IEEE Press, 2002. [Online.] Available: http:// www-mobile.ecs.soton.ac.uk

[20] R. D. Schafer, An Introduction to Nonassociative Algebras. New York: Dover, 1996.

[21] T. Moon and W. Stirling, Mathematical Methods and Algorithms for Signal Processing. Upper Saddle River, NJ: Prentice Hall, 2000.

[22] H. Holma and A. Toskala, eds., WCDMA for UMTS : Radio Access for Third Generation Mobile Communications. Chichester, UK: John Wiley and Sons, Ltd., 2000.

[23] S. Bug, C. Wengerter, I. Gaspard, and R. Jakoby, "Channel model based on comprehensive measurements for DVB-T mobile applications," in Proc. IEEE Instrumentation Measurements Technol. Conf., May 2001, vol. 1, pp. 544-548.

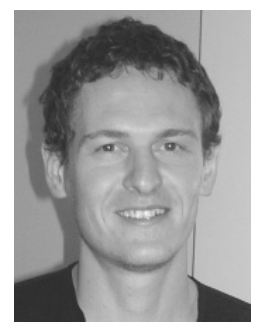

Jos Akhtman (M'00) received the B.Sc. degree in Physics and Mathematics in 2000 from the Hebrew University of Jerusalem, Israel. From 2000 to 2002 he was working as a Research Engineer in VYYO Ltd., Jerusalem, Israel. He is currently working towards the Ph.D. degree in the Communications Group, School of Electronics and Computer Science at the University of Southampton, UK. His major subject of interest is optimization algorithms for advanced multi-antenna multi-carrier communication systems. Specifically, iterative channel estimation, detection, space-time processing and turbo transceiver architecture.

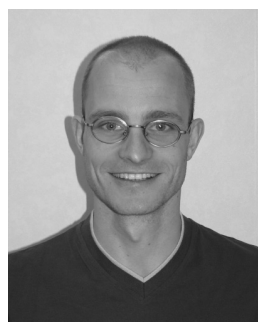

Andreas Wolfgang (M'03) was born in Germany, in 1977. He received the Dipl. Ing. degree in electrical engineering from Karlsruhe University of Technology, Karlsruhe, Germany, in 2003, and is currently pursuing the Ph.D. degree at the University of Southampton, Southampton, U.K. His primary research interests include wideband communication systems, iterative detection as well as nonlinear signal processing.

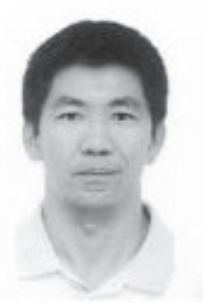

Sheng Chen (M'90-SM'97) obtained a BEng degree from the East China Petroleum Institute, Dongying, China, in 1982, and a $\mathrm{PhD}$ degree from the City University, London, in 1986, both in control engineering. In 2005, he was awarded the DSc from the University of Southampton, Southamptom, UK. Since 1999 he has been with the School of Electronics and Computer Science, the University of Southampton, UK. He previously held research and academic appointments at the Universities of Sheffield, Edinburgh and Portsmouth, all in the UK.

Professor Chen's recent research works include adaptive signal processing, wireless communications, modelling and identification of nonlinear systems, neural network and machine learning, finite-precision digital controller design, evolutionary computation methods, and optimization. He has published over 280 research papers. In the database of the world's most highly cited researchers in various disciplines, compiled by Institute for Scientific Information (ISI) of the USA, Prof. Chen is on the list of the highly cited researchers in the engineering category (see http://www.ISIHighlyCited.com). 

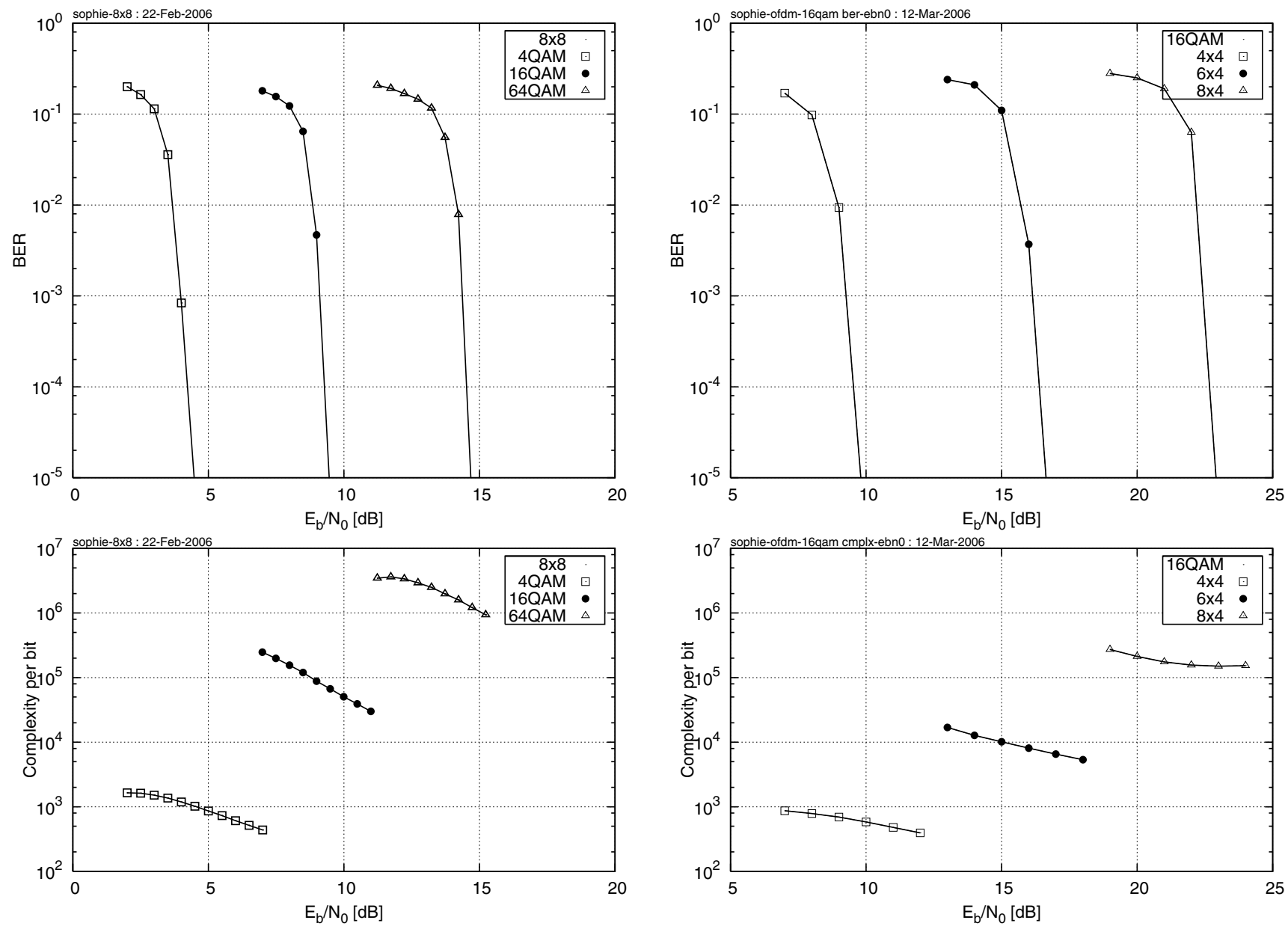

(a)

(b)

Fig. 4. Bit Error Rate (top) and the associated computational complexity per detected bit (botom) exhibited by the SDM-OFDM system employing the SOPHIE SDM detector of Algorithm 2 and assuming $\rho=1.3, \gamma=0.8$. (a) $8 \times 8$ system employing 4, 16 and 64 QAM, and (b) 16QAM system employing a fixed number of 4 receive antennas, as well as 4,6 and 8 transmit antennas. The abscissa represents the average $E_{b} / N_{0}$ recorded at the receive antenna elements.

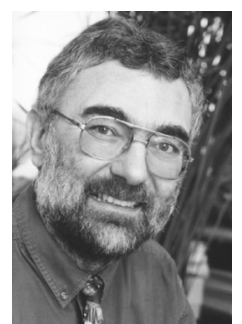

Lajos Hanzo (M'91-SM'92-F'04) Fellow of the Royal Academy of Engineering, received his firstclass degree in electronics in 1976 and his doctorate in 1983. In 2004 he was awarded the Doctor of Sciences (DSc) degree by the University of Southampton, UK. During his career in telecommunications he has held various research and academic posts in Hungary, Germany and the UK. Since 1986 he has been with the Department of Electronics and Computer Science, University of Southampton, UK, where he holds the chair in telecommunications. He co-authored 15 books totalling 10,000 pages on mobile radio communications, published in excess of 700 research papers, organised and chaired conferences, presented various keynote and overview lectures and has been awarded a number of distinctions. Currently he heads an academic research team, working on a range of research projects in the field of wireless multimedia communications sponsored by industry, the Engineering and Physical Sciences Research Council (EPSRC) UK, the European IST Programme and the Mobile Virtual Centre of Excellence (VCE), UK. He is an enthusiastic supporter of industrial and academic liaison and he offers a range of industrial courses. Lajos is also an IEEE Distinguished Lecturer of both the Communications as well as the Vehicular Technology Society, and is a Fellow of both the IEEE and the IEE. He is an editorial board member of the PROCEEDINGS OF THE IEEE and a Governor of the IEEE VT Society. For further information on research in progress and associated publications please refer to http://wwwmobile.ecs.soton.ac.uk http://www-mobile.ecs.soton.ac.uk 